\title{
96. RECORD OF THE MARINE CYANOBACTERIA FROM THE ROCKY SHORES OF BET-DWARKA AND OKHA, INDIA
}

\author{
Vishal SHAH, Nikki GARG and Datta MADAMWAR
}

Citas de cianobacterias marinas del litoral rocoso de Bet-Dwarka y Okha, India

Keywords. Cyanobacteria, Sea Shore, Flora, Bet-Dwarka, Okha, India

Palabras clave. Cianobacteria, litoral marino, flora, Bet-Dwarka, Okha, India

Cyanobacteria - the largest group of phototropic prokaryotes have been in the corner stone of biology for long. They are oxygenic, photosynthetic, microorganisms and are widely distributed over a diverse range of habitats (Nagarkar, 1998). Remaining in the oblivion, uncared and unrecognized, it has shot into fame and popularity owing to a host of their innate properties that make them ideal organisms for use in a variety of ways to meet our needs and to promise us a bright future. Presently the biotechnological exploitations range from its use in production of biofuel such as hydrogen (Bagai \& Madamwar, 1999); wastewater treatment (Shah et al., 1999); production of antimicrobial compounds (Smith \& Doan, 1999); exopolysaccharides (Shah et al., 2000) and other various applications in fertilizer and food industry. At this juncture of expansion of cyanobacterial biology it is essential to explore new species of cyanobacteria existing in the nature, isolate and purify it and subsequently establish a collection, which then could be a door for the biotechnological exploitation (Shah et al, 2000). Komarek, \& Anagnostidis (1986) reported the new approach for the classification of cyanophytes.

As described in detail (Thajjudin \& Subramanian, 1992) very little work has been carried out on the cyanobacterial flora of marine areas of India. Also, with the exceptionally high rate of species getting extinct, the study on the distribution of types of species across different distributions is necessary. The regular study on such lines will help us to know the effect of anthropogenic activities on the colonization and decolonization of the species at particular locus.

Extensive literature survey has shown no records of cyanobacteria from the shores of Bet-Dwarka and Okha, India. Therefore an attempt has been made in this direction.

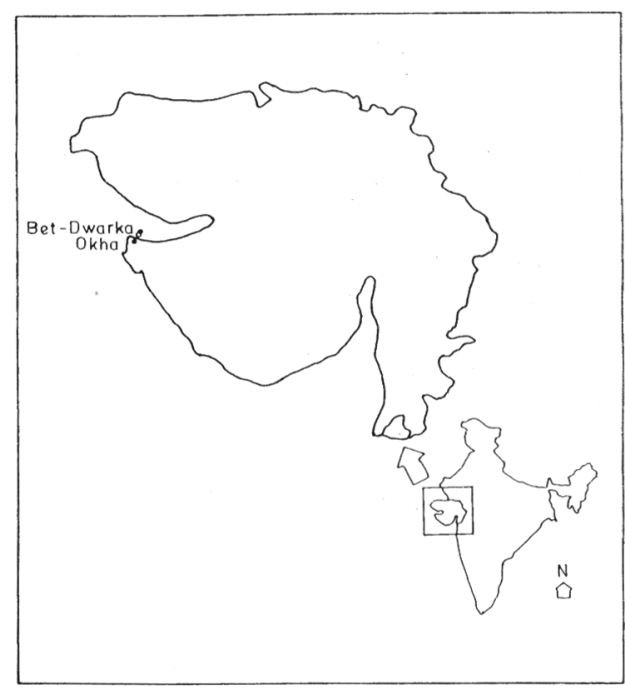

Figure 1. Location of the study area at Gujarat, India. 
Samples of cyanobacteria were collected from the rocky shores of Bet-Dwarka and Okha ports, Gujarat, India (located between 20' and 25 ' latitude and 65' and 70' longitude) (fig. 1). The cultures were collected from shore sand, stones, floating biomass and other substrates along the sea. The cyanobacteria specimens were collected in polythene bags and plastic vials and were later transferred to sterile seawater media having $100 \mathrm{mg} \mathrm{NaNO}, 100 \mathrm{mg}$ $\mathrm{CaCl}_{2}$ and $100 \mathrm{mg} \mathrm{KH}_{2} \mathrm{PO}_{4}$ per litre of sea water. Cyanobacteria were isolated by dilution plate and surface plating techniques. Cyanobacteria were made free of diatoms and green algae by adding $0.16 \mathrm{mM}$ of cyclohexamide final concentration for 24 hours with incubation in light. Antibiotic combination of ampicillin and streptomycin was used at a final concentration of $40 \mathrm{mg} / \mathrm{ml}$ and $100 \mathrm{mg} / \mathrm{ml}$ respectively to make it free of the bacterial flora. The cultures were maintained in the modified sea water as mentioned earlier at $25^{\circ} \mathrm{C}$ with dark/light cycle of $8 / 16$ hours. The light intensity was of 3,000 lux.

Identification of the taxa was done with the help of classical manuals (Geitler 1932; Desikachary, 1959 and Fremy 1929, 1933) and observing under light microscope (40 X magnification).

\section{CYANOBACTERIA}

\section{CHROOCOCCALES}

\section{Chroococcaceae Nageli}

\section{Microcystis Kutzing}

M. litoralis (Hansg) Forti (fig. 2.a) (=Aphanocapsa litoralis Hausg.)

(Desikachary, 1959 p. 85, Geitler, 1932 p. 134; Fremy, 1933 p. 11)

Colony round or ellipsoidal, with colonial mucilage that is not homogenous, cells spherical or ellipsoidal, many in single colony, with distinct individual sheaths, up to twice as long as broad, closely arranged, pale blue-green.
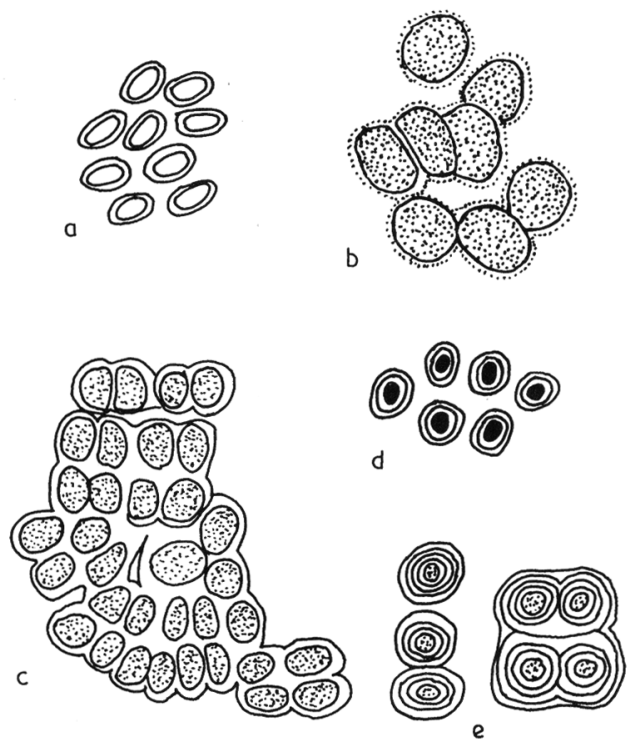

Figure 2. (a) Microcystis litoralis (Hansg) Forti (b) Chrococcus minutus (Kutz) Nag (c) Chrococcus cohaerens (Breb). Nag (d) Chrococcus westii (W. West) Boye-Petersen (e) Gleocapsa decorticans (A. Br.) Richter.

\section{Chroococcus Nag}

Ch. minutus (Kutz) Nag (fig. 2. b)

(Desikachary, 1959 p.102, pl. 24, fig. 4 \& pl. 26, figs. 4, 15; Geitler 1932, p. 232, fig. $112 \mathrm{a}, 113 \mathrm{c}$; Fremy 1933, p.24, pl. 4, fig. 6)

Cells are spherical; single rarely in groups of 2-4, diameter of 4-6 $\mu \mathrm{m}$ (with out sheath); cell content homogenous; colonies 10-12 $\mu \mathrm{m} \mathrm{X} \mathrm{15-17}$ $\mu \mathrm{m}$; light blue green.

\section{Ch. cohaerens (Breb). Nag (fig. 2.c)}

(Desikachary 1959, p. 111, pl. 26, fig. 3,9; Geitler 1932, p. 238, fig. 116 c; Fremy 1929, p. 44, fig. 47)

Thallus slimy, gelatinous, blue or dark-green; cells single or up to $2-8$ in group, without envelope 4-7 $\mu$ m diameter, with sheath $5-7 \mu \mathrm{m}$ diameter; sheath thin, colorless, unlamellated.

Ch. westii (W. West) Boye-Petersen (fig. 2.d)

(Desikachary 1959, p. 103; Geitler 1932, p. 230, fig. 108d) 

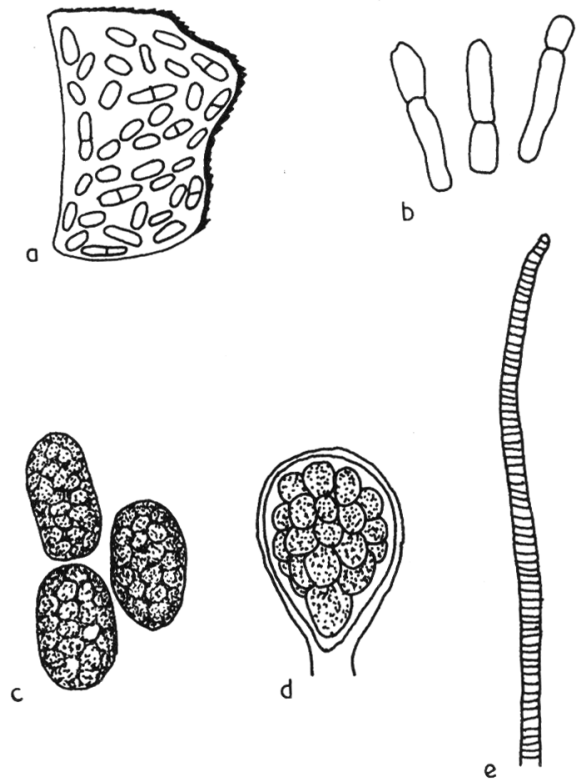

Figure 3. (a) Aphanothece microscopica Nag. (b) Synechococcus elongatus Nag (c) Synechococcus aeruginosus Nag. (d) Dermocarpa leibleiniae (Reubscg) Bornet et Thuret (e) Oscilatoria laetevirens (Crouan) Gomont Var. minimus Biswas.

Cells single or in groups of 2-4, without sheath 13-27 $\mu$ m diameter, with sheath 18-32 $\mu$ m diameter, violet; sheath colourless, distinctly lamellated.

\section{Gleocapsa Kutzing}

G. decorticans (A. Br.) Richter (fig. 2.e)

(Desikachary 1959, p. 114, pl. 24, fig. 9; Geitler 1932, p.184, fig. 83b)

Cells spherical or sometimes oval, blue-green, single or up to 2-4 together; single cells with 19 X 21 $\mu \mathrm{m}$, without sheath $6 \times 8 \mu \mathrm{m}$, in a two celled stage with sheath $22 \times 30 \mu \mathrm{m}$, without sheath up to $12 \mu \mathrm{m}$ long; sheath colorless, thick, distinctly lamellated.

\section{Aphanothece Nag.}

A. microscopica Nag. (fig. 3.a)

(Desikachary 1959, p. 142, pl. 22, fig. 4,5,9; Fremy 1929, p 28, fig. 30; Geitler 1932, p. 172, fig. 79).

Thallus small gelatinous, amorphous, up to 2 $\mathrm{mm}$ diameter; cells oblong, cylindrical, more or less $4.5 \mu \mathrm{m}$ broad, $11 / 2-2$ times as long as broad, with sometimes distinct, individual sheath, bluegreen, colorless, nannocytes present.

\section{Synechococcus Nag.}

\section{S. elongatus Nag. (fig. 3.b)}

(Desikachary 1959, p. 143, pl. 25, fig. 7,8; Geitler 1932, p. 273, fig. 133a-c)

Cells cylindrical, $1.4-2 \mu \mathrm{m}$ broad, $11 / 2-3$ times as long as broad, single or 2-4 cells together; contents homogenous and light blue green.

\section{S. aeruginosus Nag. (fig. 3.c) (=Cyanothece aeruginosus) \\ (Desikachary 1959, p. 143, pl. 25, fig. 6,12;} Geitler 1932, p. 274, fig. 133d)

Cells cylindrical, $5-16 \mu \mathrm{m}$ broad, up to $30 \mu \mathrm{m}$ long, single, or 2-4 together, pale blue-green.

\section{CHAMAESIPHONALES}

\section{Dermocarpaceae}

\section{Dermocarpa Crouan}

D. leibleiniae (Reinsch) Bornet et Thuret (fig. 3.d)

(Desikachary 1959, p. 173, pl.33, fig. 20-21; Geitler 1932, p. 399, fig. 224; Fremy 1933, p. 161, pl. 17, fig. 3)

The sporangia is olive-green or brownish, mostly single, elongate, oval or spherical, $7-9 \mu \mathrm{m}$ broad, with a thick dark brown membrane. The entire protoplasm is divided successively to form 6 - 19 endospores which are $1-2 \mu \mathrm{m}$ in diameter.

\section{OSCILLATORIALES}

\section{Oscillatoriaceae}

\section{Oscillatoria Vaucher}

O. laetevirens (Crouan) Gomont var. minimus Biswas (fig. 3.e)

(Desikachary 1959, p. 213, pl. 39, fig. 2,3)

Trichomes $2.5-3 \mu \mathrm{m}$ in diameter, somewhat fragile, slightly constricted at the cross walls, apex of the trichome slightly tapering, more or less curved, not distinctly hooked, apical cell acute and 
somewhat pointed, calyptra absent; cells $1.5-2 \mu \mathrm{m}$ in length; cross walls granulated, 3 granules on either side; cell contents uniformly granular, blue-green.

\section{Trichodesmium Ehrenb.}

T. erythraeum Ehrenberg ex Gomont (fig. 4.a)

(Desikachary 1959, p. 245, pl. 42, fig. 1,2; Geitler 1932, p.968, figs. 617a,d)

Trichomes in free swimming bundles, straight, parallel, constricted at the cross-walls, the ends gradually attenuated, $7-11 \mu \mathrm{m}$ broad, rarely upto 21 $\mathrm{mm}$; cells as long as broad or up to $1 / 2$ as long as broad, $5.4-11 \mu \mathrm{m}$ long; apex with a depressed conical or convex calyptra.

\section{Phormidium Kutz.}

Ph. corium (Ag.) Gomont (fig. 4.b)

(Desikachary 1959, p. 269, pl.44, fig. 10-11; Geitler 1932, p. 1018, fig. 649; Fremy 1929, p. 150, fig. 133)

Thallus expanded, membranous, leathery; filaments are olive green, straight, densely entangled; sheath is diffluent, hyaline and very thin; trichomes are $1-3 \mu \mathrm{m}$ wide with slightly constricted cross walls; cells are barrel shaped; slightly shorter than wide; cross walls not granulated; the end cells are obtuse conical.

\section{Ph. tenue (Menegh) Gomont (fig. 4.c)}

(Desikachary 1959, p 259, pl 43, fig. $13-15$ $\&$ pl 44, fig. 7a; Geitler 1932, p 1004, fig. 642 d,e; Fremy 1929, p. 146, fig. 131)

Thallus pale blue-green, thin, membranous, expanded; trichome straight or slightly bent, densely entangled, slightly constricted at cross walls, attenuated at the ends, $1-2 \mu \mathrm{m}$ broad, pale bluegreen; sheath thin; cells up to 3 times longer than broad, end cells acute conical.

\section{Ph. fragile (Meneghini) Gomont (fig. 4.d)}

(Desikachary 1959, p 253, pl 44, fig. 1-3; Geitler 1932, p 999, fig. 636 b; Fremy 1933, p. 86, pl. 22, fig. 6)

The filaments are dull blue green, more or less flexuous entangled or nearly parallel; sheath is hyaline and very thin; the trichomes are $1-2 \mu \mathrm{m}$ wide, constricted cell cross walls; cells are quadrate or longer than wide; $1-3 \mu \mathrm{m}$ long; cross walls are not granulated and the protoplasm is homogenous. The end cells are attenuated, acute conical without a calyptra.
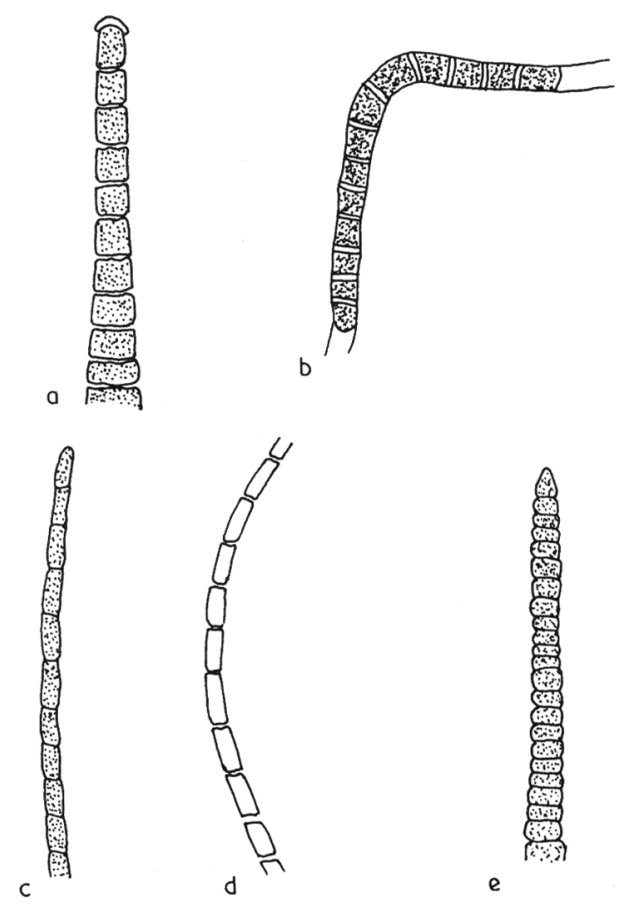

Figure 4. (a) Trichodesmium erythraeum Ehrenbergy ex Gomont (b) Phormidium corium (Ag.) Gomont (c) Phormidium tenue (Menegh) Gomont (d) Phormidium fragile (Meneghine) Gomonot (e) Phormidium jadinianum Gomont.

\section{Ph. jadinianum Gomont (fig. 4.e)}

(Desikachary 1959, p 256, pl 55, fig. 9; Geitier 1932, p 1002, fig. 640; Fremy 1929, p. 136, fig. 118).

Thallus dark-green, thin, amorphous; filaments more or less parallel; sheath thin, diffluent; trichome olive-green, distinctly constricted at the cross-walls, with straight long acuminate ends, $4-6 \mathrm{~mm}$ broad; cells shorter than broad to nearly quadrate, $2-3.5$ $\mathrm{mm}$ long, contents granulated with a hyaline central area, septa not granulated; end cell acute conical, calyptra absent.

\section{Lyngbya Ag.}

L. limnetica Lemmermann (fig. 5.a)

(Desikachary 1959, p 294, pl 50, fig. 11; Geitler 1932, p 1046, fig. 661 a,b; Fremy 1933, p. 

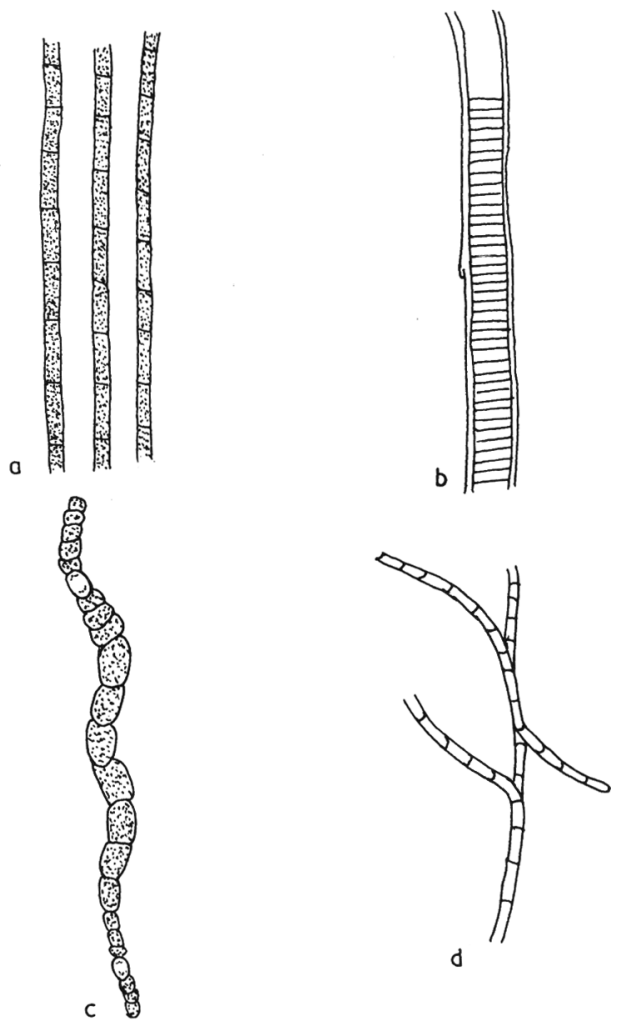

Figure 5. (a) Lyngbya limnetica Lemmermann (b) Lyngbya martensiana Menegh. ex Gomont (c) Anabena variabilis Kutzing ex Born. Et Flah (d) Plectonema terbrens Bornet ex Gomont.

\section{0,pl. 29, fig. 3)}

Filaments straight or slightly curved or coiled, single, free-floating, 1-2 $\mu \mathrm{m}$ broad; sheath thin, colourless; cells $1-1.5 \mu \mathrm{m}$ broad, quadrate to $1 / 3$ rarely $1 / 8$ as long as broad, $1-3 \mu \mathrm{m}$ long, not constricted at the cross walls, with or without a granule at the cross-walls, pale blue-green; end cells not attenuated, rounded.

\section{L. martensiana Menegh. ex Gomont (fig. 5.b)}

(Desikachary 1959, p 318, pl 52, fig. 6; Geitler 1932, p 1064, fig. 676; Fremy 1933, p. 107,pl. 29, fig. 1).

Thallus caespitose, blue-green, when dried violet, filaments long more or less flexible; sheath colourless, thick; trichome $6-10 \mathrm{~mm}$ broad, not constricted at the cross walls, cross wall sometimes granulated, apices not attenuated, pale blue-green; cells $1 / 2-1 / 4$ times as long as broad, $1.75-3.3 \mu \mathrm{m}$ in length; end cell rounded, without calyptra.

\section{Plectonema Thuret}

P. terebrans Bornet ex Gomont (fig. 5.d)

(Desikachary 1959, p 435, pl 61, fig. 4,5; Geitler 1932, p 683, fig. 437 a; Fremy 1933, p. 99 , pl. 25, fig. 5).

Filaments long, flexuous, with sparse false branching; false branches single, sheath very thin, trichomes blue-green, not constricted at the crosswalls; cells are $2-6 \mu \mathrm{m}$ long, granule on either side of the cross-walls, end cells rounded.

\section{NOSTOCALES}

\section{Nostocaceae}

\section{Anabena Bory}

A. variabilis Kutzing ex Born. Et Flah (fig. 5.c) (= Trichormus variabilis)

(Desikachary 1959, p 410, pl 71, fig. 5; Geitler 1932, p 876, fig. 558; Fremy 1929, p. 360, fig. 294)

Thallus gelatinous, dark-green; trichome without any sheath, flexuous, $4-6 \mu \mathrm{m}$ broad, slightly constricted at the cross-walls, end cells conical, obtuse; cells barrel-shaped, sometimes with gas-vacuoles, $2.5-6 \mu \mathrm{m}$ long; heterocysts spherical or oval, $6 \mu \mathrm{m}$ broad, up to $8 \mathrm{~mm}$ long; akinetes formed centrifugally, not contiguous with the heterocysts, barrel-shaped, in series, $7-9 \mu \mathrm{m}$ broad, $8-14 \mu \mathrm{m}$ long, epispore smooth, or with fine needles, colourless.

ACKNOWLEDGEMENT. The work was sponsored by University Grants Commission, New Delhi.

\section{REFERENCES}

BAGAI, R. \& D. MADAMWAR - 1999- Long-term photo-evolution of hydrogen in a packed bed reactor containing a combination of Phormidium valderianum, Halobacterium halobium and Escherichia coli immobilized in polyvinyl alcohol. International Journal of Hydrogen Energy 24, 311 - 317. 
DESIKACHARY, T.V. -1959- Cyanophyta. Indian Council of Agricultural Research, New Delhi. $686 \mathrm{pp}$.

FREMY, P. -1929- Les Myxophycees de l'Afrique equatoriale francaise. Arch. Bot. Caen 3 : Memoire no.2.

FREMY, P. -1933- (reprinted 1972)- Cyanophyces des Cotes d'Europe Mem. Soc. Natn. Sci. Nat. Math. Cherbourg 41: 1-236.

GEITLER, L. -1932- Cyanophyceae In: Rabenhorst's Kryptogamen flora. Akademische Verlagsgesellschaft Lepzig. 1196 pp.

KOMAREK, J. \& K. ANAGNOSTIDIS -1986Modern approach to the classification system of cyanophytes. 2.-Chroococcales. Archives Hydrobiology. Suppl. 73, 2, Algological Studies 43: 157-226.

NAGARKAR, S. -1998- New records of marine cyanobacteria from Rocky shores of Hong Kong. Botanica Marina 41:527 - 542.

SHAH, V., N. GARG \& D. MADAMWAR -1999Exopolysaccharide production by a marine cyanobacterium Cyanothece sp. Application in dye removal by its gelation phenomenon. Applied Biochemistry and Biotechnology 82, 81 - 90 .
SHAH, V., N. GARG \& D. MADAMWAR -2000Charaterization of the extracellular polysaccharide produced by a marine cyanobacterium Cyanothece sp and its application toward metal removal from solutions. Current Microbiology 40: 274-278.

SHAH, V., N. GARG \& D. MADAMWAR -2000Record of the cyanobacteria present in the Hamisar pond of Bhuj, India. Acta Bot. Malacitana 25:175-180.

SMITH, G.D. \& N.T. DOAN -1999- Cyanobacterial metabolites with bioactivity against photosynthesis in cyanobacteria, algae and higher plants. Journal of Applied Phycology 11: 337-344.

THAJUDDIN, N. \& G. SUBRAMANIAN -1992Survey of cyanobacterial flora of the southern east coast of India. Botanica Marina 35 : 305 - 314.

Aceptado para su publicación en julio de 2001

Author's addresses. Post Graduate Department of Biosciences. Sardar Patel University. Vallabh Vidyanagar 388 120. Gujarat. India.

\title{
97. NOTAS COROLÓGICAS DEL MACROFITOBENTOS DE ANDALUCÍA (ESPAÑA). V
}

\author{
José Carlos BÁEZ, Francisco CONDE y Antonio FLORES-MOYA
}

New records for the macrophytobenthos of Andalusia (Spain). V.

Palabras clave. Andalucía, Asparagopsis taxiformis, Desmarestia dresnayi, Macroalgas marinas, Spatoglossum solierii.

Key words. Andalusia, Asparagopsis taxiformis, Desmarestia dresnayi, Spatoglossum solierii, seaweeds.

El listado de macroalgas marinas del litoral andaluz ha sido reseñado por FloresMoya et al. (1995a, 1995b) y Conde et al. (1996a, 1996b). Este trabajo continua en esa línea, aportando 14 nuevas citas para las provincias de Almería, Cádiz, Granada y 